\title{
A clinical tool to measure plagiocephaly in infants using a flexicurve: a reliability study
}

This article was published in the following Dove Press journal:

Pediatric Health, Medicine and Therapeutics

3 October 2013

Number of times this article has been viewed

\section{Amy Leung' \\ Pauline Watter ${ }^{2}$ \\ John Gavranich ${ }^{3}$}

'Department of Physiotherapy, Royal Children's Hospital, Brisbane, Australia; ${ }^{2}$ Physiotherapy Division, University of Queensland, Brisbane, Australia; ${ }^{3} \mathrm{Child}$ and Family Health Services, West Moreton Health Service District, Ipswich, Australia
Correspondence: Amy Leung University of Queensland, PO Box 2396, Runcorn, Brisbane, QLD 4II3, Australia Tel +61732190196 Fax+61732190196 Email amyleungpt@gmail.com
Purpose: There has been an increasing incidence of infants presenting with plagiocephaly in the last two decades. A practical, economical, and reliable clinical plagiocephaly measure is essential to assess progression and intervention outcomes. This study investigated the reliability of a modified cranial vault asymmetry index using a flexible curve in infants.

Measurement: A flexicurve was molded to the infant's head and its shape maintained as it was placed onto paper to trace the head shape. Using a small modification of Loveday and De Chaplain's procedure to measure a cranial vault asymmetry index, a pair of diagonals were drawn at $30^{\circ}$ through the midpoint of the central line to their intersection with the traced head outline. The difference in length of the paired diagonals was divided by the short diameter then multiplied by $100 \%$, yielding the modified cranial vault-asymmetry index.

Patients and methods: Infants referred to a community health physiotherapist for assessment due to suspected abnormal head shape were included. To explore intrarater reliability, 34 infants aged 3-14 months were measured twice $\left(\mathrm{T}_{1} / \mathrm{T}_{1}{ }^{\prime}\right)$ at the beginning, and 21 of these remeasured twice at the end $\left(\mathrm{T}_{2} / \mathrm{T}_{2}{ }^{\prime}\right)$ of their physiotherapy sessions. Test-retest reliability used matchedaverage data $\left(\mathrm{T}_{1} / \mathrm{T}_{1}{ }^{\prime}\right)$ and $\left(\mathrm{T}_{2} / \mathrm{T}_{2}{ }^{\prime}\right)$ from 21 infants. To explore interrater reliability, 18 healthy infants aged 2-6 months were recruited. Each infant was measured once by each rater.

Results: For intrarater reliability, the intraclass correlation coefficient with 54 degrees of freedom $\left(\mathrm{ICC}_{\mathrm{df5} 4}\right.$ ) was 0.868 (95\% confidence interval [CI] 0.783-0.921); for test-retest reliability, $\mathrm{ICC}_{\mathrm{df20}}=0.958(95 \%$ CI $0.897-0.983)$; and for interrater reliability, $\mathrm{ICC}_{\mathrm{df17}}=0.874(95 \%$ CI 0.696-0.951).

Conclusion: The modified cranial vault asymmetry index using flexicurve in measuring plagiocephaly is a reliable assessment tool. It is economical and efficient for use in clinical settings. Keywords: plagiocephaly, modified cranial vault asymmetry index, infant, community health, reliability

\section{Introduction}

In the past two decades, there has been rising concern worldwide about the increased incidence of abnormal head shape in young infants. A dramatic increase of referrals to craniofacial specialists regarding obvious abnormal head shape was reported after the introduction of the Back to Sleep campaign in 1992. ${ }^{1,2}$ These abnormal head shapes are classified as plagiocephalic, brachycephalic, or combined. ${ }^{3}$ Brachycephaly is described as a wide-shaped head with the flat spot in the middle of the occiput. Plagiocephaly refers to an asymmetrical head shape where a flat spot occurs on one side of the occiput, with most cases caused by positional molding. ${ }^{4,5}$ The term "positional plagiocephaly" (PP) is used to differentiate this presentation from cranial asymmetry caused by craniosynostosis. In addition, compensatory changes often occur at the forehead, 
ears, and facial features, such that asymmetry throughout the face is also noticeable. ${ }^{6}$ Management of PP is conservative, and includes repositioning and cranial orthotic therapy. ${ }^{7-13}$ In primary health-care settings, infants with abnormal head shape are commonly referred to pediatric physiotherapists for advice on positioning and monitoring of the progression of their head shape. A reliable, accessible, and affordable objective measurement of cranial asymmetry is essential to guide clinical management plans.

McGarry et $\mathrm{al}^{14}$ conducted a systematic review of measurement tools measuring PP, finding that there were no standardized measurement tools that categorized the severity of the cranial asymmetry. The authors advocated that a standardized measurement technique was vital to guide appropriate treatment pathways, and that it should be reliable, noninvasive, and simple.

Cranial measurement tools reported both in research and clinical settings can be classified into medical imaging, photographic, and anthropometric methods. Medical imaging, which includes computed tomography and three-dimensional computed tomography, is used in craniosynostotic cases for presurgical preparation. ${ }^{15}$ However, concerns were raised about exposing children to excessive ionizing radiation, and it was advocated that the use of computed tomography should be limited to craniofacial deformity with uncertain diagnosis. ${ }^{16}$ The use of high-frequency ultrasound was proposed to be a safe and easy-to-use tool for confirming the diagnosis of PP. ${ }^{17,18}$ Nevertheless, sonography does not provide quantitative measurements, so its use is limited to differential diagnosis of PP from true craniosynostotic plagiocephaly. Noninvasive photographic and digital imaging have become commonly applied in research and clinical situations. Combined digital technology, use of specific computer software for analysis, and detailed cranial measurements can all be used. ${ }^{19-24}$ Studies have demonstrated that using a laser shape digitizer ${ }^{24}$ and 3-D photography ${ }^{25}$ to produce $3-\mathrm{D}$ images of the cranium are accurate and reliable. Considering the measurement procedure time and the setup cost of such systems, they may not be feasible for many busy and high-demand clinical settings.

The anthropometric measurement of cranial asymmetry can be conducted by using sliding calipers, ${ }^{26-28}$ thermoplastic material, ${ }^{20,29}$ or flexicurve. ${ }^{9}$ A reliability study using sliding calipers yielded high intrarater reliability $(\kappa=0.98)$ for asymmetry measurement and visual analysis scores $(\kappa=0.99)$, but poor interrater reliability for severity categories $(\kappa=0.28)$ and visual analysis $(\kappa=0.31) .{ }^{28}$ Wilbrand et al reported that using a sliding caliper with a standardized procedure yielded highly reproducible and reliable measurements. ${ }^{30}$ When using thermoplastic materials, van Vlimmeren et $\mathrm{al}^{29}$ demonstrated that plagiocephalometry measurements yield intrarater reliability intraclass correlation coefficients (ICCs) ranging from 0.57 to 0.97 and interrater reliability ICCs ranging from 0.65 to 0.96 . Though information will be lost when using a two-dimensional measure for a three-dimensional head shape and the measure may not represent the true asymmetry of the cranium, ${ }^{14,28}$ these tools may still suffice to guide the conservative management of infants with PP.

Loveday and de Chalain ${ }^{9}$ developed the cranial vault asymmetry index (CVAI) to measure changes in cranial asymmetry in an interventional study. The authors suggested this method was useful in clinical settings as an approximate guide to the efficacy of intervention in PP. The materials are economical, setup time is minimal, and the result can be obtained quickly. However, reliability data are lacking. This method provided the base for assessment of PP in a community health setting in Australia. Loveday and de Chalain judged the reference point on the central line for the diagonals by the intersection of the projected line joining the ears. However, the plagiocephalic head commonly has ear asymmetry, which may change as the infant grows. Thus, we used the midpoint of the central line for intersection of the diagonals on the assumption that this midpoint is consistent over time. This modification also improves practicality and efficiency of the measurement procedure. We continued to use the original formula, which yields a modified CVAI (mCVAI). This study aims to establish the intrarater reliability, test-retest reliability, and interrater reliability of the mCVAI and discuss its application in clinical settings. If reliability is established, then it would provide an alternative assessment tool for clinicians to assess cranial asymmetry, particularly when resources are limited.

\section{Methods}

\section{Design and participants}

Intrarater and test-retest reliability were evaluated retrospectively using data from a health-record audit of all infants referred to a community physiotherapist for assessment due to suspected abnormal head shape between March 2008 and April 2010. This study presents the data from those presenting with plagiocephaly only. Interrater reliability was prospectively studied, in a community health center where healthy infants were recruited for measurement between September 2012 and November 2012. Ethical approval was obtained from the human-research ethics committees of the University of Queensland and the Darling Downs-West Moreton Health Service District. 


\section{Measurements}

As described by Loveday and de Chalain, ${ }^{9}$ a flexicurve was used to obtain a circumferential head tracing. We used a small modification of their technique to attempt to improve the accuracy of this clinical measure, as described below.

For each measurement, two markings were placed on the infant's head in vertical alignment with the nasion and inion. The nasion is the central point of the frontonasal suture. ${ }^{31}$ It can be identified at the midpoint of the nose bridge, and extending vertically upwards allows the center point to be marked "N" on the forehead. This mark should be visible when the flexicurve is in position (Figure 1). The inion is the most prominent point of the external protuberance of the occiput. ${ }^{31}$ The rater can identify the inion by sliding the thumb tip along the cervical groove up to the occiput where the external occipital crest can be felt first and then the inion. The rater continued to extend the thumb tip vertically upwards, and a mark (I) was then made on the occiput. The mark should be visible above the flexicurve when it is placed on the infant's head (Figure 2). A reference mark was made on the flexicurve where the $\mathrm{N}$ was to be positioned with respect to the flexicurve. The $\mathrm{N}$ marked on the forehead was lined up with the $\mathrm{N}$ marked on the flexicurve, and the flexicurve was wrapped closely around the infant's head (Figure 3). The position of the inion could then be read off from the calibrations on the flexicurve. The lower rim of the flexicurve was aligned horizontally (in line with the Frankfurt lines) and at the maximum occipitofrontal circumference (Figure 4). The Frankfurt line is the line joining the inferior margin of the bony orbit and superior margin of the external acoustic meatuses. The Frankfurt plane, which is formed by the Frankfurt lines on either side, denotes the anatomical horizontal plane of the head. ${ }^{31}$

The flexicurve was then lifted off the head and placed on paper while its shape was carefully maintained. If there is a

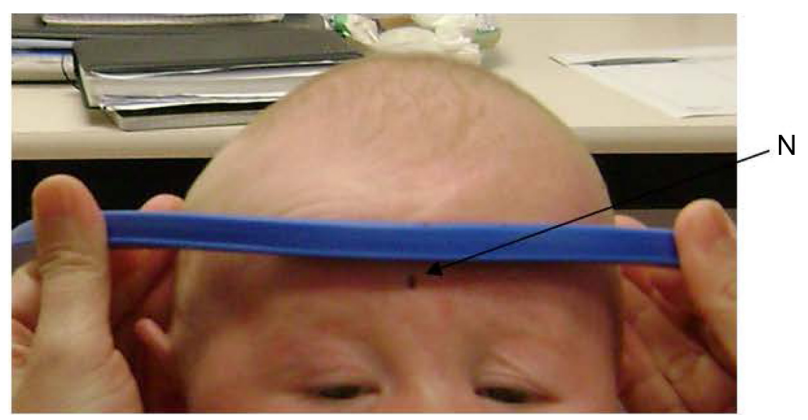

Figure I Anterior view of flexicurve placement.

Note: Align the $\mathrm{N}$ marking on flexicurve with the $\mathrm{N}$ marking on the forehead. Abbreviation: N, Nasion.

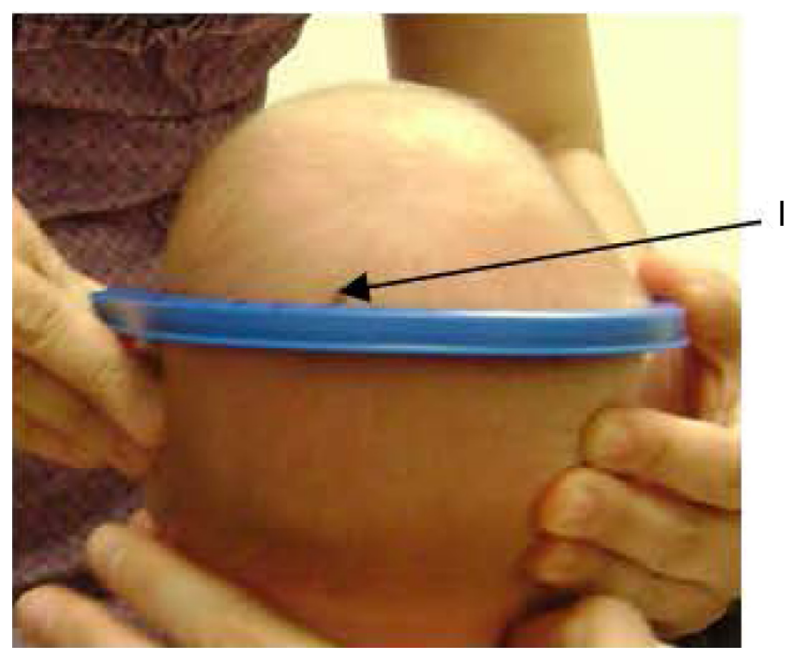

Figure 2 Posterior view of flexicurve placement.

Note: (I) marking on the occiput is visible above the flexicurve.

Abbreviation: I, Inion.

resistance to removal, that means the flexicurve is below the maximum circumference. If it slides off too easily, then the flexicurve is above the maximum circumference. The "just right" feel can be improved by practical trials on a round object or doll. Adjustment to the position is then required. The corresponding $\mathrm{N}$ and I markings on the flexicurve were aligned with the central line on the mCVAI form. The flexicurve was stabilized on the top to maintain its shape on the paper. The head circumference was traced using a sharp pencil as close as possible to the inner side of the flexicurve (Figure 5), and the midpoint of the length of the head (N-I) was marked on the central line (M). Two diagonals of $30^{\circ}$ from the central line were drawn through point $\mathrm{M}$ and their

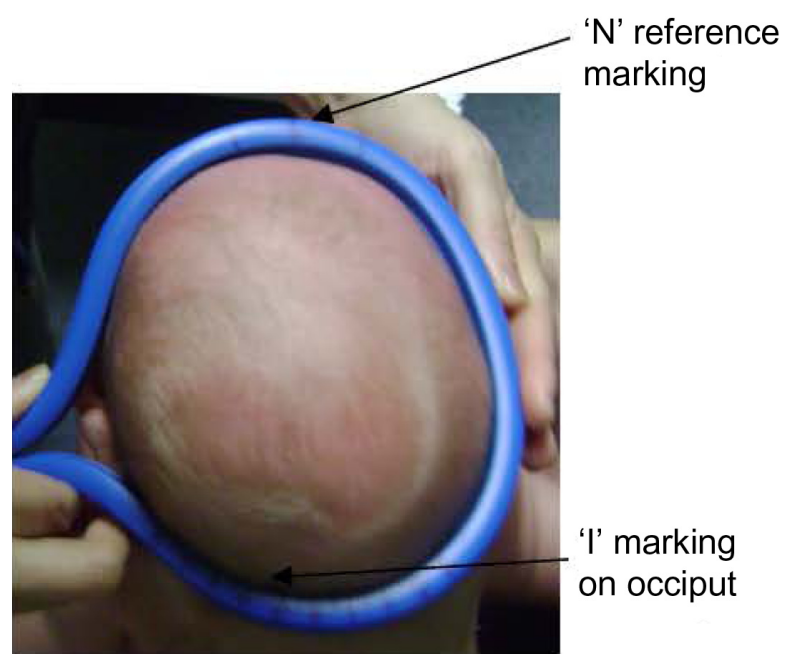

Figure 3 Top view of flexicurve placement.

Note: The flexicurve is wrapped around the head with the ends locking on the side. Abbreviations: N, Nasion; I, Inion. 


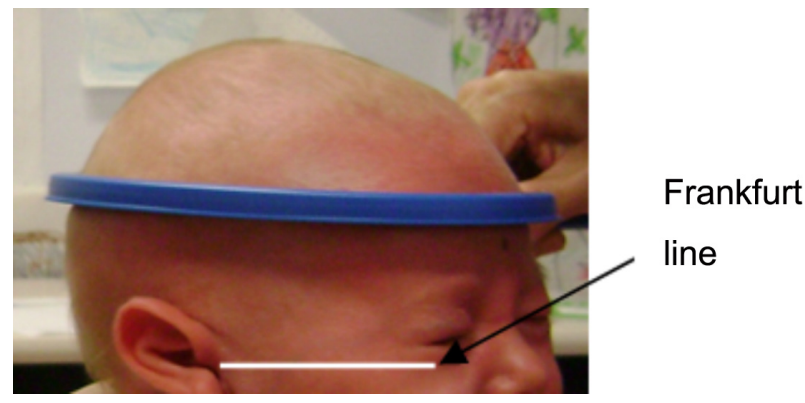

Figure 4 Side view of flexicurve placement.

Note: The flexicurve is align with the Frankfurt line.

lengths were measured (Figure 6). As per Loveday and de Chalain, ${ }^{9}$ the mCVAI was calculated as follows:

$$
\text { CVAI }=\frac{\text { Short diagonal }- \text { Long diagonal }}{\text { Short diagonal }} \times 100 \%
$$

A perfectly symmetrical head should have a CVAI score of $0 \%$, while a head is considered to have significant asymmetry if the CVAI is $>-3.5 \%$. However, it was not stated clearly in the original article how this value was determined. In other studies, various cutoff points and classification of severity reported in plagiocephaly measurements were based on clinical experience, parental concerns, and clinical perception. ${ }^{20,29,32}$ Wilbrand et al measured a group of 401 infants to obtain normative values of cranial vault growth in the first year of life. ${ }^{3}$ The anthropometric measurements of infants referred to their specialist clinics with nonsynostotic abnormal head shape were compared with these normative data. The authors proposed that the classification of positional head deformities and severity of the deformity should be

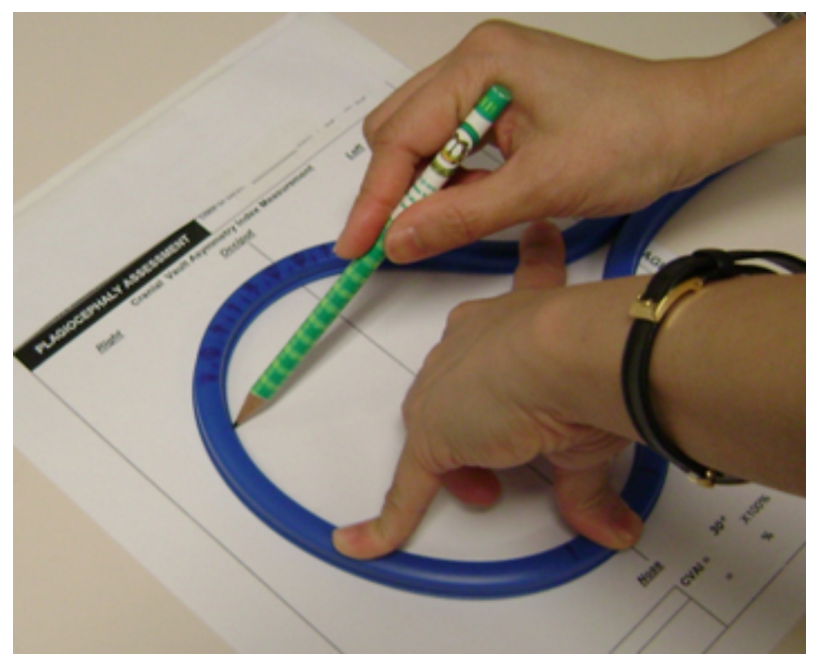

Figure 5 Tracing of head shape on the CVAI form.

Note: Tracing the head shape at the inner rim of the flexicurve with a sharp pencil.

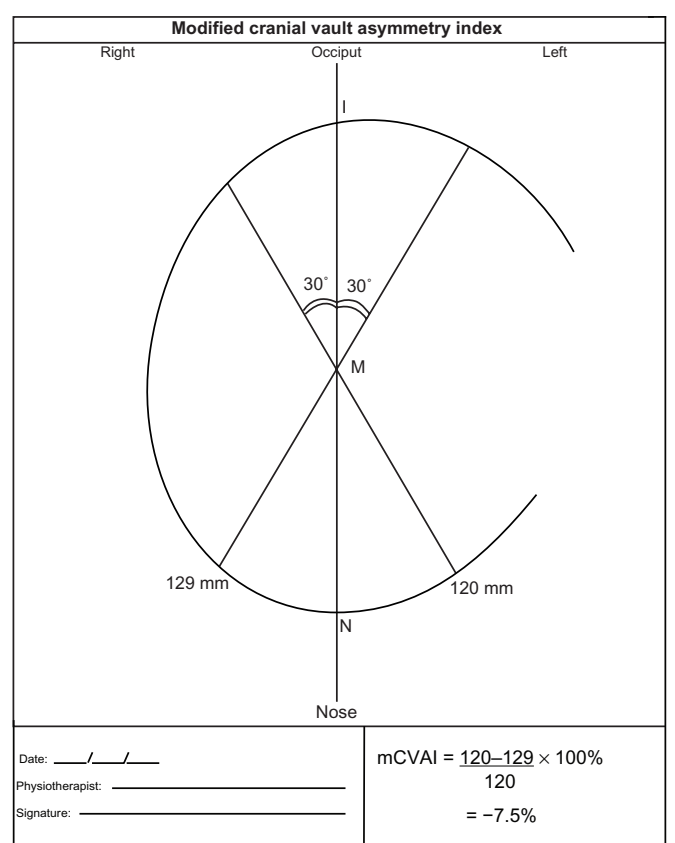

Figure $6 \mathrm{mCVAl}$ tracing and calculation.

Note: The head tracing on the mCVAl form with markings and diagonal lines.

Abbreviations: N, Nasion; I, Inion; M, mid-point of central line; mCVAI, modified Cranial Vault Asymmetry Index.

norm-referenced according to age, sex, and country. This approach of collecting normative data when using standardized measurement should be adopted.

\section{Equipment}

Materials required were a $60 \mathrm{~cm}$-long flexicurve (Celco, New Taipei City, Taiwan, ROC) which is made of a strip of malleable rubber. This brand was selected due to its flexibility in molding on the infant's head and rigidity in holding the shape once molded. Since there are no markings on the flexicurve, calibrations were drawn on the top surface of the flexicurve to provide reference points for ease of measurement. Other materials required included a colored fine-point pen (0.4) to make markings on the infant's head, a sharp pencil (or Pacer pencil) for marking the head tracing, an angle ruler to measure the angle of the diagonals, a ruler to draw the diagonals and measure the length of the diagonals, and a calculator for calculation of the index. A customized mCVAI recording form was developed in which a line was drawn in the middle to represent the central line of the cranium.

\section{Procedure}

\section{Intrarater and test-retest reliability study}

The mothers provided informed consent for the measurement procedure at the beginning of the session. All measurements were conducted by the same physiotherapist. Infants were 
measured twice $\left(\mathrm{T}_{1} / \mathrm{T}_{1}{ }^{\prime}\right)$ at the beginning of the session, and then underwent their usual physiotherapy intervention. The second set of measurements $\left(\mathrm{T}_{2} / \mathrm{T}_{2}{ }^{\prime}\right)$ was performed at the end of the session for those infants available for testretest assessment. The time between the initial and repeated measures ranged from 40 to 50 minutes. The calculation of the mCVAI was completed only at the end of the session; therefore, the therapist had no prior knowledge of the mCVAI results when remeasurements occurred.

The infants were mostly in a calm and content state during measurements. The infant was positioned sitting sideways on the mother's lap and facing the mother's right side, while the rater stood in front of the infant. The mother used her right hand to support the infant's chin and her left hand to support the base of the occiput. The flexicurve was applied as previously described. The rater held and secured both ends of the flexicurve, and this was referred as its locking point. When locking the flexicurve, gentle force only was applied to avoid discomfort to the infant. To leave the $\mathrm{N}$ and I points clear for observation, the locking point should be at the side of the head. The calibration corresponding to the I point was noted, and the flexicurve was then carefully removed and transferred to the mCVAI form without altering its shape. The mCVAI was calculated according to the procedure described previously.

\section{Interrater-reliability study}

Consent was gained for the measurement procedure. The infant was measured once by each rater. The author was rater 1 (R1), and a physiotherapist from the community health service was rater 2 (R2). Prior to the study, R2 received training in mCVAI measurement from $\mathrm{R} 1$, who is experienced in using the measurement method. The order of the raters conducting the measurements was alternated to avoid order bias.

\section{Data analysis}

Statistical analysis was performed using SPSS version 19 (IBM, Armonk, NY, USA). All infants were measured twice at the beginning $\left(\mathrm{T}_{1} / \mathrm{T}_{1}{ }^{\prime}\right)$, and available infants were again measured twice $\left(\mathrm{T}_{2} / \mathrm{T}_{2}{ }^{\prime}\right)$ at the end of the session. The repeated measurements, ie, $T_{1} / T_{1}{ }^{\prime}$ and $T_{2} / T_{2}{ }^{\prime}$, were used to evaluate intrarater reliability. The matched averages of $\mathrm{T}_{1} / \mathrm{T}_{1}{ }^{\prime}$ and $\mathrm{T}_{2} / \mathrm{T}_{2}{ }^{\prime}$ measures were used to evaluate test-retest reliability. The interrater reliability was calculated using R1 and R2 data. The data were analyzed using ICCs, with alpha set at $P=0.05$. Levels of reliability used were from Domholdt, ${ }^{33}$ where very high was $0.9-1$, high was $0.7-0.89$, and moderate was $0.5-0.69$.

\section{Results}

\section{Intrarater reliability}

Thirty-four data sets of $\mathrm{T}_{1} / \mathrm{T}_{1}{ }^{\prime}$ and 21 data sets of $\mathrm{T}_{2} / \mathrm{T}_{2}{ }^{\prime}$ measurements were extracted, providing 55 data sets for intrarater-reliability analysis. The infants' mean age $( \pm$ standard deviation [SD]) was $26 \pm 12.22$ weeks, and median age was 24 weeks and ranged from 11.57 to 62.14 weeks. The sex ratio was 24 males to ten females. Nineteen infants had right-side flatness, and 15 had left-side flatness. There was neither torticollis nor syndromic diagnosis among these infants. The mean value for mCVAI at initial measurement $( \pm$ SD) was $5.71 \% \pm 2.22 \%$, and at immediate remeasurement was $5.80 \% \pm 2.09 \%$. Intrarater reliability was established with ICC with 54 degrees of freedom $\left(\mathrm{ICC}_{\mathrm{df5} 4}\right)=0.868(P=0.001$, 95\% confidence interval $[\mathrm{CI}] 0.783-0.921)$.

\section{Test-retest reliability}

Twenty-one sets of matched-average $T_{1} / T_{1}{ }^{\prime}$ and $T_{2} / T_{2}{ }^{\prime}$ were available for test-retest reliability analysis. The mean value for mCVAI at initial measurement $( \pm \mathrm{SD})$ was $5.54 \% \pm 2.21 \%$, and at later remeasurement was $5.64 \% \pm 2.29 \%$. Test-retest reliability for average measures was established with $\mathrm{ICC}_{\mathrm{d} 20}=0.958(P=0.001,95 \%$ CI $0.897-0.983)$.

\section{Interrater reliability}

A total of 18 infants were measured by both examiners. The infants' mean age $( \pm \mathrm{SD})$ was $16.01 \pm 5.8$ weeks, and median age was 14.87 weeks and ranged from 9 to 26.71 weeks. The sex ratio was seven males to eleven females. The mean value for mCVAI measured by R1 ( \pm SD) was $1.00 \% \pm 3.20 \%$, and $\mathrm{R} 2$ was $1.24 \% \pm 2.78 \%$. Interrater reliability: was established with $\mathrm{ICC}_{\mathrm{df17}}=0.874(95 \% \mathrm{CI} 0.696-0.951)$. There were two data sets with clear rater-recording errors, where R1 and R2 recorded a different side of flatness.

\section{Discussion}

This study is the first step in providing evidence that the mCVAI is a reliable clinical tool to measure plagiocephaly, which would allow monitoring of treatment outcomes. The mCVAI measured using the flexicurve was reliable across applications for an experienced clinician and for a trained novice clinician. The practicality of using the mCVAI measurement in clinical settings is supported by its accuracy and time efficiency, and by economic considerations.

It is clear that there are some factors that may impact on the accuracy of the measurement. As pointed out by Mortenson and Steinbok, ${ }^{28}$ infant behavior and identification of landmarks might contribute to errors in the measurement. 
Firstly, it is very difficult to obtain a correct head mold of the flexicurve when the infant is struggling and unsettled. Therefore, the infant should always be in a settled state before measurement is attempted. Secondly, care should be taken to recheck the alignment of the extended points from the original nasion and inion to improve accuracy. Thirdly, if the infant has a lot of hair, there may be added difficulty in molding the flexicurve close to the head, and the inion marking on the head may not be easily visible. Fourthly, using a blunt pencil to trace the circumference will generate measurement error. ${ }^{9}$ Fifthly, the flexicurve should be replaced when its shape cannot be maintained. Repeated practice will refine the rater's skills, and we suggest that practice is essential to achieve measurement accuracy.

The time needed to conduct the measurement, including preparation and process time, is under 10 minutes, which is consistent with Hutchison et al's study. ${ }^{23}$ The flexicurve needs to be on the infants' head only briefly, and this enhances compliance of the infant with the measurement procedure, contributing to the feasibility of the tool. The mCVAI can be calculated at any time in a clinic session, and provides immediate feedback to the parents about the severity of the asymmetry, whereafter a management plan can be discussed.

The materials required by this measurement tool can be obtained from stationery stores, and are easily portable from clinic to clinic. In addition, no extra resources are required to obtain the results. In contrast, other measurement methods use expensive digital cameras or computer software for which funding may not be available in clinical settings or in home visits. The authors suggest that the mCVAI method is not limited to the clinic, but may also be used in home environments, making it particularly useful for outreach services to remote and rural areas. Another application of this tool could be to generate a cephalic index, which is a measurement of brachycephalic head shape, but this is beyond the scope of this paper.

Though Loveday and de Chalain ${ }^{9}$ commented that this method may not have adequate precision in research studies, this study showed that it is useful in clinical settings, having high test-retest reliability as well as high intrarater reliability, when used by careful raters trained in this method. This enhances the continuum of care when the infant is being seen by multiple physiotherapists. Further work is needed to explore the effect of using different cutoff points to categorize the severity of PP. The validity of this measurement is warranted to show strong correlation with clinical observatory judgement. ${ }^{14,28}$ Normative data from the mCVAI will enhance the determination of cutoff points and categorization of the severity of the deformities.

\section{Limitations}

Using the $30^{\circ}$ diagonal either side of the midline may not capture the maximum asymmetry present. For example, the asymmetry may be more significant at the side for a more brachycephalic head compared with dolichocephalic head shape. However, this may not be a significant factor, because the same points are used in the infant over time. In addition, this could also explain the discrepancy in subjective assessment of the parents and objective assessment by the clinician. From clinical experience, it is not uncommon to hear that parents indicate improvement in the infants' head shape, although this may not be demonstrated by objective measurements.

The time gap between $T_{1}$ and $T_{2}$ measurement should ideally be longer, as a clinician's memory may make it easier to place the flexicurve at the same position after a short time gap. Preferably, a few days' separation of testing times would have enhanced the study. However, in clinical practice, the infants commonly had monthly appointments, and remeasuring after a month's gap would be complicated by growth of the head.

\section{Conclusion}

Measurement of cranial asymmetry expressed by the mCVAI using a flexicurve is a reliable, practical, and economical measurement tool for use in primary care community health settings by experienced and novice physiotherapists. This provides a suitable tool for physiotherapists' use in the clinical management of infants presenting with PP. It may be particularly useful to those providing outreach services in remote and rural areas where resources are scarce.

\section{Acknowledgments}

The authors would like to thank Timothy Effeney for being the rater in assisting the interrater-reliability study. The authors would also like to give a special thanks to the Therapy and Support Service for Children team for providing the resources for this study to be conducted.

\section{Disclosure}

The authors report no conflicts of interest in this work.

\section{References}

1. Argenta LC, David LR, Wilson JA, Bell WO. An increase in infant cranial deformity with supine sleeping position. J Craniofac Surg. 1996;7: $5-11$. 
2. Turk AE, McCarthy JG, Thorne CH, Wisoff JH. The "back to sleep campaign" and deformational plagiocephaly: is there cause for concern? J Craniofac Surg. 1996;7:12-18.

3. Wilbrand JF, Schmidtberg K, Bierther U, et al. Clinical classification of infant nonsynostotic cranial deformity. Journal Pediatr. 2012;161: 1120-1125.

4. O’Broin ES, Allcutt D, Earley MJ. Posterior plagiocephaly: proactive conservative management. Br J Plast Surg. 1999;52:18-23.

5. Littlefield TR, Kelly KM, Reiff JL, Pomatto J. Car seats, infant carriers, and swings: their role in deformational plagiocephaly. J Prosthet Orthot. 2003; 15:102-106

6. Argenta LC. Clinical classification of positional plagiocephaly. J Craniofac Surg. 2004;15:368-372.

7. Ripley CE, Pomatto J, Beals SP, Joganic EF, Manwaring KH, Moss SD. Treatment of positional plagiocephaly with dynamic orthotic cranioplasty. J Craniofac Surg. 1994;5:150-159.

8. Graham JM, Lucas BC. Helmet treatment for plagiocephaly during infancy. Pediatr Res. 1997;41:60.

9. Loveday BP, de Chalain TB. Active counterpositioning or orthotic device to treat positional plagiocephaly? J Craniofac Surg. 2001;12: 308-313.

10. de Chalain T. The Safe-T-Sleep device: safety and efficacy in maintaining infant sleeping position. $N$ Z Med J. 2003;116:U581.

11. Bialocerkowski AE, Vladusic SL, Howell SM. Conservative interventions for positional plagiocephaly: a systematic review. Dev Med Child Neurol. 2005;47:563-570.

12. Elwood ET, Petronio J, Wood RJ. Parental satisfaction with the craniocap: a new cranial orthosis for deformational plagiocephaly. Cleft Palate Craniofac J. 2005;42:340-343.

13. Lee WT, Richards K, Redhed J, Papay FA. A pneumatic orthotic cranial molding helmet for correcting positional plagiocephaly. J Craniofac Surg. 2006;17:139-144.

14. McGarry A, Dixon MT, Greig RJ, Hamilton DR, Sexton S, Smart H. Head shape measurement standards and cranial orthoses in the treatment of infants with deformational plagiocephaly. Dev Med Child Neurol. 2008;50:568-576.

15. Frühwald J, Schicho KA, Figl M, Benesch T, Watzinger F, Kainberger F. Accuracy of craniofacial measurements: computed tomography and three-dimensional computed tomography compared with stereolithographic models. J Craniofac Surg. 2008;19:22-26.

16. Fearon JA, Singh DJ, Beals SP, Yu JC. The diagnosis and treatment of single-sutural synostoses: are computed tomographic scans necessary? Plast Reconstr Surg. 2007;120:1327-1331.

17. Sze RW, Parisi MT, Sidhu M, et al. Ultrasound screening of the lambdoid suture in the child with posterior plagiocephaly. Pediatr Radiol. 2003;33:630-636.

18. Regelsberger J, Delling G, Tsokos M, et al. High-frequency ultrasound confirmation of positional plagiocephaly. J Neurosurg. 2006;105: 413-417.
19. Panchal J, Marsh JL, Park TS, Kaufman B, Pilgram T. Photographic assessment of head shape following sagittal synostosis surgery. Plast Reconstr Surg. 1999;103:1585-1591.

20. Chang PY, Chien YW, Huang FY, Chang NC, Perng DB. Computeraided measurement and grading of cranial asymmetry in children with and without torticollis. Clin Orthod Res. 2001;4:200-205.

21. Littlefield TR, Kelly KM, Cherney JC, Beals SP, Pomatto JK. Development of a new three-dimensional cranial imaging system. J Craniofac Surg. 2004;15:175-181.

22. Zonenshayn M, Kronberg E, Souweidane M. Cranial index of symmetry: an objective semiautomated measure of plagiocephaly. $J$ Neurosurg. 2004;100:537-540.

23. Hutchison BL, Hutchison LA, Thompson JM, Mitchell EA. Quantification of plagiocephaly and brachycephaly in infants using a digital photographic technique. Cleft Palate Craniofac J. 2005;42: 539-547.

24. Geil MD, Smith A. Accuracy and reliability of a system for the digital capture of infant head shapes in the treatment of cranial deformities. J Prosthet Orthot. 2008;20:35-38.

25. Schaaf H, Pons-Kuehnemann J, Malik CY, et al. Accuracy of threedimensional photogrammetric images in non-synostotic cranial deformities. Neuropediatrics. 2010;41:24-29.

26. Moss SD. Nonsurgical, nonorthotic treatment of occipital plagiocephaly: what is the natural history of the misshapen neonatal head? JNeurosurg. 1997;87:667-670.

27. Mulliken JB, Vander Woude DL, Hansen M, LaBrie RA, Scott RM. Analysis of posterior plagiocephaly: deformational versus synostotic. Plast Reconstr Surg. 1998;103:371-380.

28. Mortenson PA, Steinbok P. Quantifying positional plagiocephaly: reliability and validity of anthropometric measurements. J Craniofac Surg. 2006;17:413-419.

29. van Vlimmeren LA, Takken T, van Adrichem LN, van der Graaf Y, Helders PJ, Engelbert RH. Plagiocephalometry: a non-invasive method to quantify asymmetry of the skull; a reliability study. Eur J Pediatr. 2006;165:149-157.

30. Wilbrand JF, Wilbrand M, Pons-Kuehnemann J, et al. Value and reliability of anthropometric measurements of cranial deformity in early childhood. J Craniomaxillofac Surg. 2011;39:24-29.

31. Drake RL, Vogl AW, Mitchell AW. Gray's Anatomy for Students. 2nd ed. Philadelphia: Churchill Livingstone/Elsevier; 2010:813-816, 1062.

32. Hutchison BL, Mitchell EA, Thomson JM. Non-synostotic plagiocephaly and brachycephaly: an overview. Curr Pediatr Rev. 2006;2:33-39.

33. Domholdt E. Rehabilitation Research: Principles and Applications. 3rd ed. Philadelphia: Elsevier Saunders; 2004.
Pediatric Health, Medicine and Therapeutics

\section{Publish your work in this journal}

Pediatric Health, Medicine and Therapeutics is an international, peerreviewed, open access journal publishing original research, reports, editorials, reviews and commentaries. All aspects of health maintenance preventative measures and disease treatment interventions are addressed within the journal. Practitioners from all disciplines are invited to submit

\section{Dovepress}

their work as well as healthcare researchers and patient support groups. The manuscript management system is completely online and includes a very quick and fair peer-review system. Visit http://www.dovepress.com/ testimonials.php to read real quotes from published authors. 